\title{
Loss and Dynamic Magnetic Field Measurements in LHC Dipoles
}

\author{
A. Akhmetov, S. Amet, I. Balaazi, L. Bottura, M. Gateau, P. Pugnat, and L. Walckiers
}

\begin{abstract}
Knowledge of $\mathrm{AC}$ loss and dynamic magnetic field distortion in the main LHC dipoles is both important for the assessment of the accelerator performance and providing insight into the properties of assembled magnets. We measured the loss due to the current cycling in a few 1-meter long model dipoles, 15-meter long dipole prototypes and pre-series magnets. As expected the loss depends linearly on the rate of the current change. From the slope of this dependence, the contact resistance between the strands of the opposite layers of the cable, $R_{c}$, was evaluated for the inner winding of the dipole. We discuss the method to estimate the $R_{c}$ value in the outer winding. The $R_{c}$ value has been also derived independently from measurements of the magnetic field. For this, the ramp rate dependent component of the main field as well as of the harmonics has been measured. The main magnetic field measurements were performed using both stationary coils and Hall probes. Rotating coils were used to perform the harmonic measurements.
\end{abstract}

Index Terms-AC loss, coupling currents, dipole magnet, magnetic field quality, superconducting cables.

\section{INTRODUCTION}

$\mathbf{S}$ UPERCONDUCTING strands and cables subjected to a changing magnetic field exhibit effects related to persistent currents in superconducting filaments and normal currents flowing between both the filaments of each strand and the strands of the cable. These currents disturb the background magnetic field and dissipate the energy provided by the magnetic field source. In accelerator dipole magnets wound with Rutherford-type superconducting cables, the magnetic field distortion is the main issue that should be addressed, for instance, by proper synchronization of the power converters.

The present paper is based on measurements conducted in 1999-2001. We measured the loss and dynamic magnetic field distortion in a few 1-meter long model dipoles, 15-m long dipole prototypes and pre-series dipoles. All these magnets are of the six-block design shown in Fig. 1. The results are used for the evaluation of the contact electrical resistance $R_{c}$ between the crossing strands of the two layers of the cable. The last value governs the current distribution in the flat cable subjected to a time-dependent magnetic field [1], [2]. Due to the wide variety of the magnets and a gradual development of experimental techniques, we do not intend here to characterize every dipole mea-

Manuscript received September 24, 2001.

A. Akhmetov is with the CERN, European Organization for Nuclear Research, CH-1211 Geneva 23, Switzerland (LHC division, MTA group) and was with the Institute for High Temperatures, Ac. of Science of Russia, Izhorskaya 13/19, Moscow, 127412, Russia.

S. Amet, I. Balaazi, L. Bottura, M. Gateau, P. Pugnat, and L. Walckiers are with the CERN, European Organization for Nuclear Research, CH-1211 Geneva 23, Switzerland (LHC division, MTA group).

Publisher Item Identifier S 1051-8223(02)03424-3.

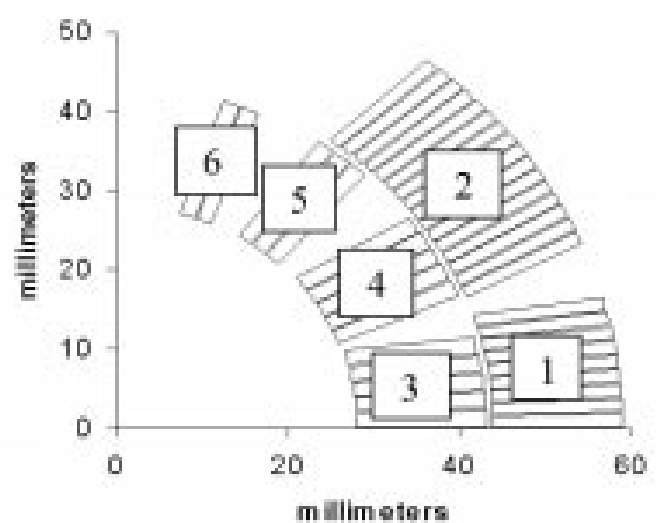

Fig. 1. Upper right quarter of the winding for one aperture of the dipole. The blocks are numbered.

sured. We will rather discuss the possibilities and restrictions of different kinds of measurements for determination of $R_{c}$ and dipole properties in general.

\section{MeAsurements TeChniQues}

All measurements are conducted during a trapezoidal current cycle from $I_{\min }$ to $I_{\max }$ and back lasting a total time $t_{c}$. The current ramps up and down are executed with constant ramp rates $a=d I / d t$ and $a=-d I / d t$ correspondingly. The total loss $W$ was obtained by recording the current $I$ and the total voltage $V$ applied to the magnet as

$$
W=\int_{0}^{t_{c}} V I d t
$$

In addition to the total voltage we measured voltage drops in portions of the magnet. In this case (1) provides an apparent loss in the portion of the magnet measured.

The magnetic field harmonics $B_{n}$, where $n=1$ stands for the main field, have been measured during the up and down ramps. As the result we obtained the values

$$
\Delta B_{n}=\frac{1}{2}\left[B_{n}\left(I_{m}, a\right)-B_{n}\left(I_{m},-a\right)\right],
$$

where $I_{m}=\left(I_{\min }+I_{\max }\right) / 2$. Measurements of $B_{1}$ were done by the stationary coils [3], [4] and the Hall probe setup described in [5]. Rotating coils [6] were used for the evaluation of the higher order harmonics at the reference radius $17 \mathrm{~mm}$. The experimental techniques are described in more detail in [3]-[7]. 


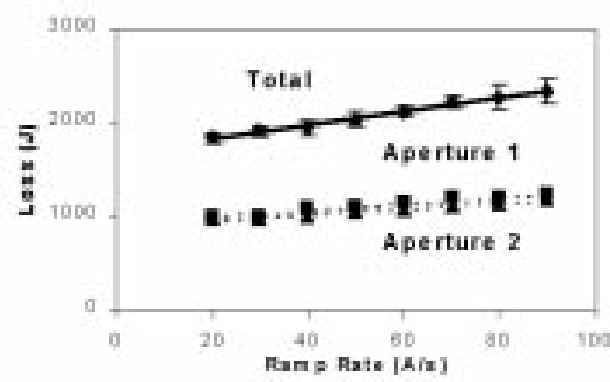

Fig. 2. Loss in the pre-series dipole MBPSO01.

\section{LOSS MEASUREMENTS}

\section{A. Total Loss and Its Scaling}

Fig. 2 shows the results of loss measurements conducted on the Alstom-made pre-series dipole MBPSO01 for $I_{\min }=1000$ A and $I_{\max }=4000$ A. Each point in this figure corresponds to the average of five measurements, the error bars show standard deviations. In spite of the fact that the same conditions were used for the measurements, the points exhibit substantial scatter. This is probably due to the deviation of the performed current cycle from the ideal one. Nevertheless, it is seen that the dependence $W=W(d I / d t)$ can be fitted by the linear expression:

$$
W=W_{h}+K a,
$$

where $W_{h}=1.7 \cdot 10^{3} \mathrm{~J}$ and $K=7.4 \mathrm{Js} / \mathrm{A}$. The corresponding standard deviations are $\sigma\left(W_{h}\right)=80 \mathrm{~J}$ and $\sigma(K)=1.4 \mathrm{Js} / \mathrm{A}$.

The ramp rate independent component of the loss, $W_{h}$ is the hysteresis loss in the superconducting filaments and, to a much lesser extent, in the iron yoke of the dipole. The coefficient $K$ defines the loss in the metallic elements of the construction, and among them mainly in the inter-strand contacts and in the matrix of the strands.

Experiments conducted for different $I_{\min }$ and $I_{\max }$ indicated that within the measurement accuracy the coefficient $K$ can be expressed via another coefficient $k$ as

$$
K \approx 2 \Delta I k
$$

where $\Delta I=I_{\max }-I_{\min }$. This relation can be used to compare measurements performed in different current intervals.

To evaluate the interstrand contact resistance $R_{c}$, the coefficient $k$ is corrected for the strand matrix (interfilament) contribution and the contribution from the metallic parts of the magnet, mostly copper wedges inserted between the cable blocks (see Fig. 1). The coefficient obtained after such a correction, $k_{c}$, is compared to the interstrand loss coefficient $\kappa^{c}$ calculated for the reference $R_{c}$ value by means of computer codes [8], [9]. This allows us to evaluate the contact resistance of the magnet. Calculations show that the main interstrand contribution to the total loss comes from the 3rd, 4th, and the 5 th blocks of the inner winding. Hence, the $R_{c}$ determination based on the loss measurements provides the value averaged over these three blocks.
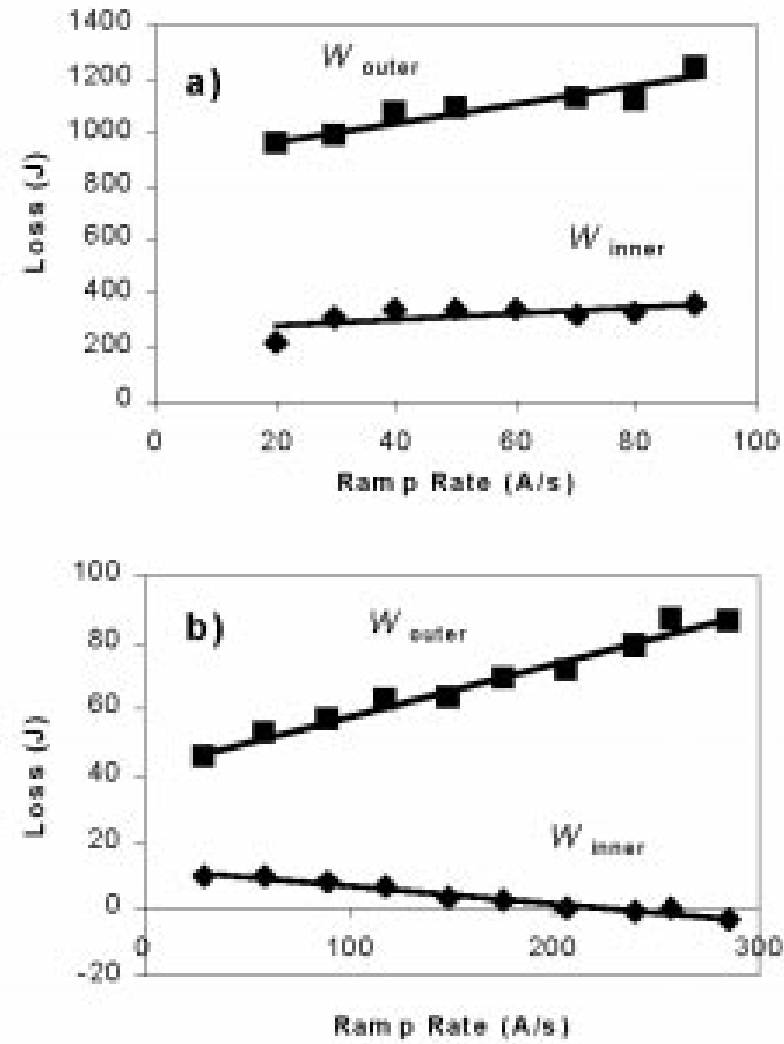

Fig. 3. Apparent loss in the inner ( $)$ and outer ( $\mathbf{\square})$ windings of the dipole; (a) the first aperture of MBP2O1 prototype, (b) the 1-m long one aperture MBSMS21 dipole. Contrary to direct calculations based on [8] and [9], the apparent loss is higher in the outer winding. The negative slope of the loss dependence for the inner winding reflects small contact resistance in the outer winding.

\section{B. Apparent Loss}

Loss measurements can be also conducted on different portions of the dipole winding thus providing the partial values of the loss. For instance, $W_{1}$ and $W_{2}$ shown in Fig. 2 characterize the loss in the first and the second aperture of the MBPSO01 dipole. Also, for a few dipoles we measured $W_{\text {up }}$ and $W_{\text {down }}$, which are the losses in the upper and lower windings of one of the apertures. In the same way, $W_{\text {inner }}$ and $W_{\text {outer }}$ are the measured losses in the inner and outer windings of the same aperture. The corresponding partial loss coefficients can be defined in accordance with (3) and (4) and denoted as $k_{\text {inner }}$ and $k_{\text {outer. }}$.

The ratio $k_{\text {inner }} / k_{\text {outer }}<1$ (see Fig. 3 ) found in the experiments with the 1-m long dipoles and dipole prototypes is in a gross contradiction with the ratio of calculated values $\kappa_{\text {inner }}^{c} / \kappa_{\text {outer }}^{c}=37.2$ [9]. In some cases, e.g., for the one-aperture dipole MBSMS21/V2, the coefficient $k_{\text {inner }}$ is negative enough to even make the apparent loss $W_{\text {inner }}$ negative at high ramp rates. A possible explanation of this effect is given in [10]. An apparent loss measured in a portion of the magnet does not coincide with the real energy deposited in it. Rather it depends on both the magnetic flux penetrating the portion in question and the local contact resistance. In the absence of inter-filament 


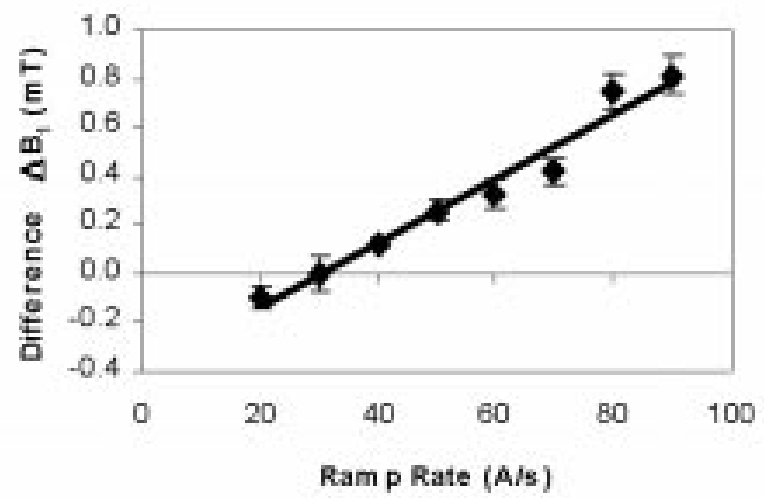

Fig. 4. The difference $\Delta B_{1}$ in the 2 nd aperture of the MBPSA01 dipole measured with stationary coils.

coupling within a strand and neglecting both hysteresis loss and contribution from the metallic parts, it can be shown that

$$
\frac{k_{\text {inner }}}{k_{\text {outer }}} \approx \frac{15 R_{c, o}-6 R_{c, i}}{110 R_{c, o}+9 R_{c, i}},
$$

where $R_{c, o}$ and $R_{c, i}$ are the contact resistances in the outer and inner windings correspondingly. It follows from (5) that $k_{\text {inner }} / k_{\text {outer }}<0$ if $R_{c, o}<0.4 R_{c, i}$. More detailed considerations including inter-filament and hysteresis loss are given in [10].

\section{Symmetry Considerations}

The magnetic flux linking the apertures of the dipole is negligible in comparison with that between the elements of one aperture. Due to this, the values $W_{1}$ and $W_{2}$ represent with good approximation the real loss in the corresponding apertures. Hence, the results shown in Fig. 2 allow us to evaluate the average $R_{c}$ in each aperture. If the loss measurement is done simultaneously in two apertures, although the absolute error is large for each aperture, the relative accuracy is still excellent. The reason is that the deviation from the ideal trapezoidal current cycle, which is the main cause for the scattering of the results, is the same for both apertures. A single measurement can be sufficient to monitor a $2 \%$ difference between $W_{1}$ and $W_{2}$. Based on the above argument, a much larger effort is needed to distinguish the origin of an asymmetry, i.e., whether it comes from the difference in the coupling currents or in the hysteresis loss.

A magnetic interaction between upper and lower halves of the dipole is very strong and the effect of the difference in $R_{c}$ on the apparent loss cannot be estimated by means of [8], [9]. However, it is clear that in an ideal dipole $W_{\text {up }}=W_{\text {down }}$. Hence, a single ramp can be used to verify the up-down symmetry of the magnet.

\section{Magnetic Field Measurements}

\section{A. $B_{1}$ Measurements With Stationary Coils}

The field measurement using stationary coils has the advantage of providing integral values along the magnet, important to assess the accelerator performance. Unluckily, it is affected by a substantial dispersion. Fig. 4 shows the results obtained for the

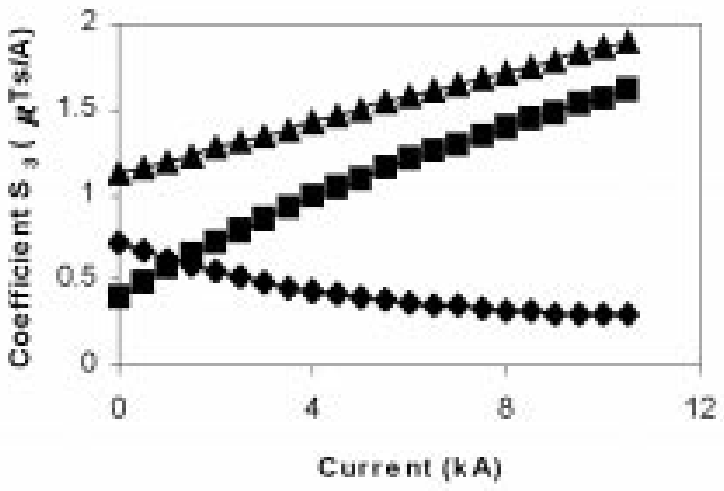

Fig. 5. The coefficient $S_{3}$ for the MBPSO01 dipole obtained in the experiment $(\boldsymbol{\square})$, calculated for the interfilament contribution $(\boldsymbol{})$ and estimated for the interstrand contribution $(\mathbf{A})$.

2nd aperture of the Ansaldo-made pre-series dipole MBPSA01. Magnetic field and loss measurements were conducted simultaneously for $I_{\min }=1000$ A and $I_{\max }=4000 \mathrm{~A}$. Each point in this figure corresponds to the average of five measurements, the error bars show standard deviations. The linear fit

$$
\Delta B_{1}=\Delta B_{1}^{(0)}+S_{1} a
$$

results in a negative offset $\Delta B_{1}^{(0)}=0.4 \mathrm{mT}, \sigma\left(\Delta B_{1}^{(0)}\right)=0.04$ $\mathrm{mT}$ consistent with the magnetic field generated by superconducting filaments. The coefficient $S_{1}=13 \mu \mathrm{T} \cdot \mathrm{s} / \mathrm{A}, \sigma\left(S_{1}\right)=$ $2.3 \mu \mathrm{T} \cdot \mathrm{s} / \mathrm{A}$ reflects mostly inter-strand coupling [8]. The current cycles were optimized for the loss measurements. Proper optimization for the magnetic field measurements could lead to a decrease in the standard deviations.

The $3 \mathrm{rd}$, the 4 th and the 5 th blocks of the inner winding are the main contributors to the difference in $B_{1}$ between the ramps up and down. Hence, for evaluation of $R_{c}$ the above data provides information equivalent to that from the loss measurements.

\section{B. $B_{1}$ Measurements with Hall Probes}

Reproducibility of $\Delta B_{1}$ measurements using Hall probes is much higher than with stationary coils. However, the results obtained with the probes located even a few centimeters apart along the magnet differ within a few percent. The presence of a periodic magnetic field pattern [11] in the dipole is mainly responsible. Another drawback of the measurements using Hall probes is that the integral magnetic field cannot be obtained directly, especially in the presence of a substantial variation of $R_{c}$ along the length of the magnet.

\section{Measurements of $\Delta B_{3}$ and $\Delta B_{5}$ With Rotating Coils}

In case of allowed harmonics, i.e., for $n=3$ (sextupole) and $n=5$ (decapole) the value $\Delta B_{n}$ was found to depend on the ramp rate $a$ as

$$
\Delta B_{n}=\Delta B_{n}^{(0)}+S_{n} a .
$$

Experiments conducted on the pre-series dipoles show that contrary to $S_{1}$, the coefficient $S_{n}$ in (7) increases with current. As an example, Fig. 5 shows the dependence of 
$S_{3}$ on current for the MBPSO01 dipole before and after a correction for the interfilament contribution done for a strand time constant $(n \tau)=0.04 \mathrm{~s}$ [12]. It is seen that the correction makes this dependence less pronounced but does not eliminate it entirely.

\section{EXAMPLES}

Let us take pre-series magnets measured so far as examples. In case of the MBPSO01 dipole, the loss (see Fig. 2) and $B_{1}$ magnetic field measurements conducted with both Hall probes and stationary coils provide averaged $R_{c} \approx 50 \mu \Omega$ for the first aperture and $R_{c} \approx 70 \mu \Omega$ for the second one. Corrections for the interfilament contributions have been done for the strand time constant $(n \tau) \approx 0.04 \mathrm{~s}$. The corrected $S_{3}$ value averaged over the current interval $I_{\min }=50 \mathrm{~A}, I_{\max }=11500 \mathrm{~A}$ is approximately $1.5 \mu \mathrm{T} \cdot \mathrm{s} / \mathrm{A}$ for the first aperture (see Fig. 5). The last value corresponds to $R_{c}=26 \mu \Omega$ [9]. An apparent contradiction between the $R_{c}$ values is, probably, due to mostly two factors. First, the $\Delta B_{3}$ value depends on the contact resistances in the blocks of the dipole in a very different way than the loss and $\Delta B_{1}$. Second, according to preliminary estimations, contribution to the $\Delta B_{3}$ from the wedges can be comparatively high and capable of screening the cable contribution.

The dipole MBPSO02 has not been studied extensively with respect to the dynamic effects in the loss and $B_{1}$. No substantial difference between the apertures has been observed either. The rough estimation of the contact resistances is $R_{c} \approx 30 \mu \Omega$ from the loss measurements and $R_{c} \approx 40 \mu \Omega$ from the $\Delta B_{1}$ measurements.

Measurements of the MBPSA01 dipole provide a good example of the symmetry check-up. While for the first aperture the loss $W_{\mathrm{up}}=W_{\mathrm{dn}}$, in the second one there is $\sim 15 \%$ difference between them. Indeed, the upper and lower poles of the outer winding of the second aperture were wound from the cables supplied by different manufacturers. With high certainty this results in the different hysteresis loss and, thus, symmetry violations.

In general, all pre-series dipoles have the coefficient $S_{1} \leq 13$ $\mu \mathrm{T} \cdot \mathrm{s} / \mathrm{A}$, much less than the upper limit for the accelerator operation. The obtained $R_{c}$ values of the order of tens of microohms are in line with the results obtained on single cables [13].

\section{CONCLUSIONS}

Of all methods discussed above none demonstrates clear advantage over the others in characterizing the dipole. The loss measurements are prone to high dispersion of the points. At the same time, even single loss measurements can be enough to assess the dipole symmetry. Besides, apparent loss measurements provide the only way to evaluate $R_{c}$ of the outer winding.

The $B_{1}$ magnetic field measurements made with stationary coils are characterized by low reproducibility but provide an information directly related to the accelerator performance. Performed with Hall probes these measurements are much more accurate locally but do not give integral values.

More information about $R_{c}$ distribution in the dipole can be obtained by harmonic measurements. Yet additional analysis has to be done to achieve consistent results in this way.

\section{ACKNOWLEDGMENT}

The authors thank S. Bouchenoua, A. Rijllart, S. Sanfilippo and T. Schreiner for their valuable help in organizing and conducting experiments.

\section{REFERENCES}

[1] A. Devred and T. Ogitsu, "Ramp-rate sensitivity of SSC dipole magnet prototypes," in Frontiers of Accelerator Technology, S. I. Kurokawa, M. Month, and S. Turner, Eds. Singapore: World Scientific, 1996, pp. 184-308.

[2] A. A. Akhmetov, "Compatibility of two basic models describing a.c. loss and eddy currents in flat superconducting cables," Cryogenics, vol. 40, pp. 445-457, 2000.

[3] J. Buckley, D. Richter, L. Walckiers, and R. Wolf, "Dynamic magnetic measurements of superconducting magnets for the LHC," IEEE Trans. Appl. Superconduct., vol. 5, pp. 1024-1027, 1995.

[4] Z. Ang, I. Bejar, L. Bottura, D. Richter, M. Sheehan, L. Walckiers, and $\mathrm{R}$. Wolf, "Measurements of AC loss and magnetic field during ramps in the LHC model dipoles," IEEE Trans. Appl. Superconduct., vol. 9, pp. 1039-1044, 1999.

[5] L. Bottura, L. Larsson, S. Schloss, M. Schneider, N. Smirnov, and M. Haverkamp, "A fast sextupole probe for snapback measurement in the LHC dipoles,", CERN-LHC-Project-Report-362, 1999.

[6] Z. Ang, L. Bottura, S. Russenschuck, A. Siemko, D. Tommasini, and L. Walckiers, "Coil size and geometic field quality in short model dipoles for LHC," IEEE Trans. Appl. Superconduct., vol. 10, pp. 53-56, 2000.

[7] A. P. Verweij, D. Leroy, L. Walckiers, and R. Wolf, "Analysis of the AC loss measurements on the one-meter dipole model magnets for the CERN LHC," IEEE Trans. Magn., vol. 30, pp. 1758-1761, 1994.

[8] L. Bottura, personal communication, CERN, LHC/MTA, Geneva, Switzerland, 2001.

[9] A. Verweij, "Print-out of the CCDI2 program," CERN, LHC/MMS, Geneva, Switzerland, 1997.

[10] A. A. Akhmetov, "Apparent energy deposition during the current cycle in the dipole magnets,", LHC-MTA-IN-2000-127, Sept. 2000.

[11] L. Bottura, L. Walckiers, and Z. Ang, "Experimental evidence of boundary induced coupling currents in LHC prototypes," IEEE Trans. Appl. Superconduct., vol. 7, pp. 801-804, 1997.

[12] R. Wolf, personal communication, CERN, LHC/MMS, Geneva, Switzerland, 2000

[13] D. Richter, personal communication, CERN, LHC/MMS, Geneva, Switzerland, 2001. 